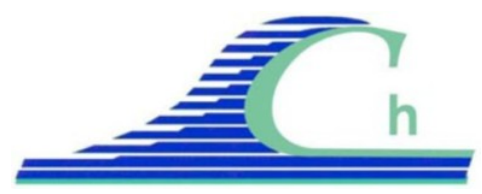

XII ${ }^{\text {èmes }}$ Journées Nationales Génie Côtier - Génie Civil

Cherbourg, 12-14 juin 2012

DOI:10.5150/jngcgc.2012.070-B @ Editions Paralia CFL

disponible en ligne - http://www.paralia.fr - available online

\title{
Gestion du patrimoine et maintenance : application de la méthode "VSC" aux ouvrages portuaires soumis à la corrosion
}

\author{
Brahim BENAISSA $^{1}$
}

1. Expert Structures au Centre d'Etudes Techniques Maritimes Et Fluviales (MEDDTL), 2, bd Gambetta, BP 60039, 60321 Compiègne, France.

b.benaissa@orange.fr

\section{Résumé :}

La gestion du patrimoine nécessite une méthode qui permet de recenser le patrimoine tout en indiquant l'état de disponibilité (fiabilité et sécurité) et d'usage de ses composantes et ensembles fonctionnels. La méthode de gestion recherchée doit s'appuyer sur la stratégie du gestionnaire pour cibler et réaliser dans l'ordre des priorités les interventions indispensables et/ou nécessaires.

Les gestionnaires des principaux ouvrages de transbordements et d'accostages sont très intéressés par le suivi de l'état de corrosion des structures métalliques et fortement demandeurs de systèmes de protection bien adaptés et optimisés en coût.

La méthode VSC (Visite Simplifiée Comparée) fait partie des démarches existantes d'aide à la gestion patrimoniale des infrastructures. Son application aux ouvrages portuaires sur la base du guide du CETMEF (2011) peut être optimisée par une approche spécifique en ce qui concerne les structures métalliques constituant les ouvrages portuaires et soumis à la corrosion. L'optimisation basée sur la maîtrise des processus de corrosion mis en jeu (principal aléa concernant les structures métalliques), permettra une application crédible de la méthode dont l'approche tend à :

i) faciliter les visites et reconnaissances sur le terrain,

ii) garantir une traçabilité et assurer un suivi des états des ouvrages.

iii)Identifier et cibler les diagnostics à réaliser avant toute opération de réparation, iv)hiérarchiser les interventions en fonction de la stratégie du gestionnaires

\section{Mots-clés :}

VSC - Etat mécaniques - Etat usage - Indice état - Indice stratégique - Patrimoine Ouvrage portuaire - Inspection - Diagnostic - Environnement maritime - Corrosion Electrochimie - Quais sur pieux - Rideaux de palplanches - Ducs d'albe

\section{Introduction}

Les gestionnaires d'ouvrages portuaires sont confrontés à un patrimoine vieillissant et hétérogène, à de nouvelles prescriptions en matière de sécurité, à la préservation de l'environnement et à la prolongation de la durée de service (AFGC, 2003). Ils doivent prendre des décisions sous contraintes économiques et ressources requises en faisant un 
choix rationnel de type protection, réparation, restauration, renouvellement ou fonctionnement dégradé.

Une approche méthodologique pour la connaissance de l'état réel des ouvrages ainsi que la priorité à donner aux interventions avec des moyens limités rend utile et nécessaire de recourir à des techniques fiables et susceptibles de fournir des éléments constituant une base de données utile pour cibler les diagnostics et choisir le mode de réparation efficace et durable (AFGC, 2005).

La méthode VSC (LCPC, 2006), comme la plupart des méthodes de gestion, nécessite au préalable de faire un recensement exhaustif des ouvrages et de disposer pour chacun d'eux d'un dossier d'ouvrage. Cet inventaire doit être structuré en prenant en compte la stratégie du maître d'ouvrage ce qui revient à affecter les ouvrages ou groupe d'ouvrages d'un indice stratégique. Celui-ci sur la base des enjeux, définit l'importance à accorder à l'état de l'ouvrage et indique le mode d'intervention à privilégier. L'indice d'état au niveau sécurité exclut cette hiérarchisation par l'importance stratégique, en effet le seul niveau de risques (combinaison aléa et vulnérabilité) peut déclencher l'intervention pour la sécurisation des personnes ou des biens. Au regard de l'indice stratégique, la nature et l'ampleur de cette intervention peut être choisi.

La méthode peut constituer un atout important dans le domaine portuaire où les contraintes d'accessibilité et d'exploitation limitent le temps de visite des ouvrages. Aussi les ouvrages sont examinés suivant trois visions parfaitement indépendantes :

- Stratégique : pour assurer en priorité l'intégrité des ouvrages à forte valeur ajoutées pour le maître d'ouvrage et optimiser l'utilisation de ses ressources.

- Mécanique : pour identifier les parties critiques des structures et évaluer les risques de dégradations connaissant leur fonctionnement, le comportement du matériau et son interaction avec le milieu maritime.

- Usage, environnement et sécurité : pour évaluer l'état de l'ouvrage en rapport avec la sécurité des usagers et l'impact sur l'environnement.

\section{La méthode VSC et la corrosion}

En bordure de mer, d'estuaire, de canal ou de rivière, des infrastructures sont réalisées et destinées pour le chargement ou le déchargement de marchandises, l'embarquement ou le débarquement de personnes et l'accostage. Des structures métalliques constituent l'ossature, le soutènement ou le support principal. Elles sont en contact avec le milieu terrestre et le milieu aquatique. Les pathologies enregistrées sur ces structures proviennent de la dégradation des métaux occasionnée par la corrosion (action chimique ou électrochimique) sous forme de perte de matière, de perforations et autres fissures et déchirures CEFRACOR (2006).

Tout en respectant l'approche des trois visions préconisée par VSC, celle mécaniques peut se définir d'une manière spécifique pour les structures métalliques soumises à la corrosion. Ce principal aléa dans un environnement maritime (embruns, marnage, 


\section{XII ${ }^{\text {èmes }}$ Journées Nationales Génie Côtier - Génie Civil \\ Cherbourg, 12-14 juin 2012}

basses eaux, immersion et mise en fiche) concerne les structures métalliques et les armatures des ouvrages en béton et se manifeste selon des zones caractéristiques avec des cinétiques variables selon les composants du milieu en contact. Ces variations de localisation et de temporalité compliquent la détection par des visites simples (sans mesures).

Une meilleure compréhension des processus mis en jeu facilite la prédétermination des zones critiques à cibler pour des inspections régulières et des diagnostics appropriés.

L'objectif de la vision mécanique est d'évaluer l'état structurel de l'ouvrage qui dépend de la résistance mécanique aux sollicitations exercées, de la qualité des matériaux principaux et leur état d'obsolescence dans la mesure où l'interaction avec le milieu provoque des dégradations suffisamment importantes pour affecter le comportement structurel. La corrosion est un processus de dégradation du métal qui se transforme en oxydes ou hydroxydes. Cette transformation affecte le matériau dans le temps avec des cinétiques variables selon des zones privilégiées. Celles-ci constituent des zones critiques et deviennent des cibles pour des visites, inspections et diagnostics régulières pour prévenir, éviter des désordres graves et éventuellement les protéger.

La méthode VSC est basée sur la réalisation de visites simplifiées et donc ciblées sur les points particuliers clairement identifiés pour chaque structure (identifiants mécaniques) Elle peut s'appliquer aisément aux structures métalliques soumises à la corrosion (figure 1).

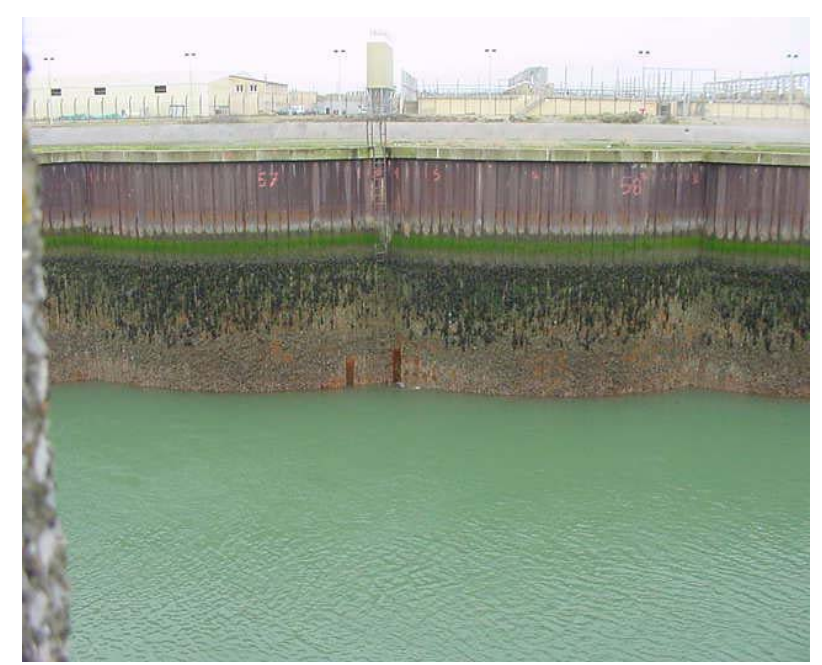

Figure 1. Illustration d'un ouvrage portuaire soumis à la corrosion.

\section{Inspection et mesures sur des structures en acier en site portuaire.}

Dans ce paragraphe nous utilisons l'exemple de suivi de la corrosion des structures métalliques en site portuaire pour indiquer l'approche rationnelle de surveillance basée sur la connaissance de l'interaction matériau - milieu en contact qui rejoint le principe de découpage et d'évaluation selon les critères de la VSC. 
Le tableau 1 indique des exemples de type d'ouvrages portuaires avec leur fonction affecté ultérieurement par le maître d'ouvrage de l'indice stratégique correspondant.

Tableau 1. Ouvrages constituant le parc.

\begin{tabular}{|c|c|c|}
\hline Ouvrages (structure et matériau) & Fonction & Indice stratégique \\
\hline $\begin{array}{l}\text { Quai sur pieux } \\
\text { (dalle en BA sur pieux en acier) }\end{array}$ & $\begin{array}{l}\text { Portance et résistance à } \\
\text { l'accostage }\end{array}$ & \\
\hline $\begin{array}{l}\text { Appontement } \\
\text { (passerelle et pieux en acier avec défenses) }\end{array}$ & $\begin{array}{l}\text { Résistance à l'accostage et } \\
\text { amarrage }\end{array}$ & \\
\hline $\begin{array}{l}\text { Ducs d'albe } \\
\text { (pieux en acier) }\end{array}$ & $\begin{array}{l}\text { Absorption d'énergie et } \\
\text { résistance aux chocs }\end{array}$ & \\
\hline Digue avec carapace (enrochement) & Protection du port & \\
\hline Forme de radoub (sas avec porte) & Carénage et mise à sec & \\
\hline Ecluse avec portes (maçonnerie et métal) & $\begin{array}{l}\text { Franchissement aux bassins } \\
\text { à flot }\end{array}$ & \\
\hline Etc & & \\
\hline
\end{tabular}

Les structures métalliques sont principalement des pieux pour les quais ou ducs d'albe et des palplanches en acier au carbone pour des rideaux de soutènement. L'assemblage des différentes parties peut nécessiter des soudures, un boulonnage ou une liaison articulée (portes et vannes). Les surfaces métalliques sont le plus souvent exposées aux embruns, au marnage ou à l'immersion sur une face et aux matériaux solides sur l'autre face.

Les quais sur pieux par exemple, sont constitués par une plate-forme supportée par des pieux (figures 2). Ils assurent les fonctions suivantes :

- reprise des efforts verticaux (charges et roulement d'outillage, charges liées à l'exploitation) par le système de pieux liaisonnés par des poutres et une dalle en béton armé,

- reprise des efforts horizontaux des navires (accostage, amarrage) ou du milieu naturel (houle ou clapotis, courant, vent),

- soutènement des terres par empiètements du talus (cas des quais). 


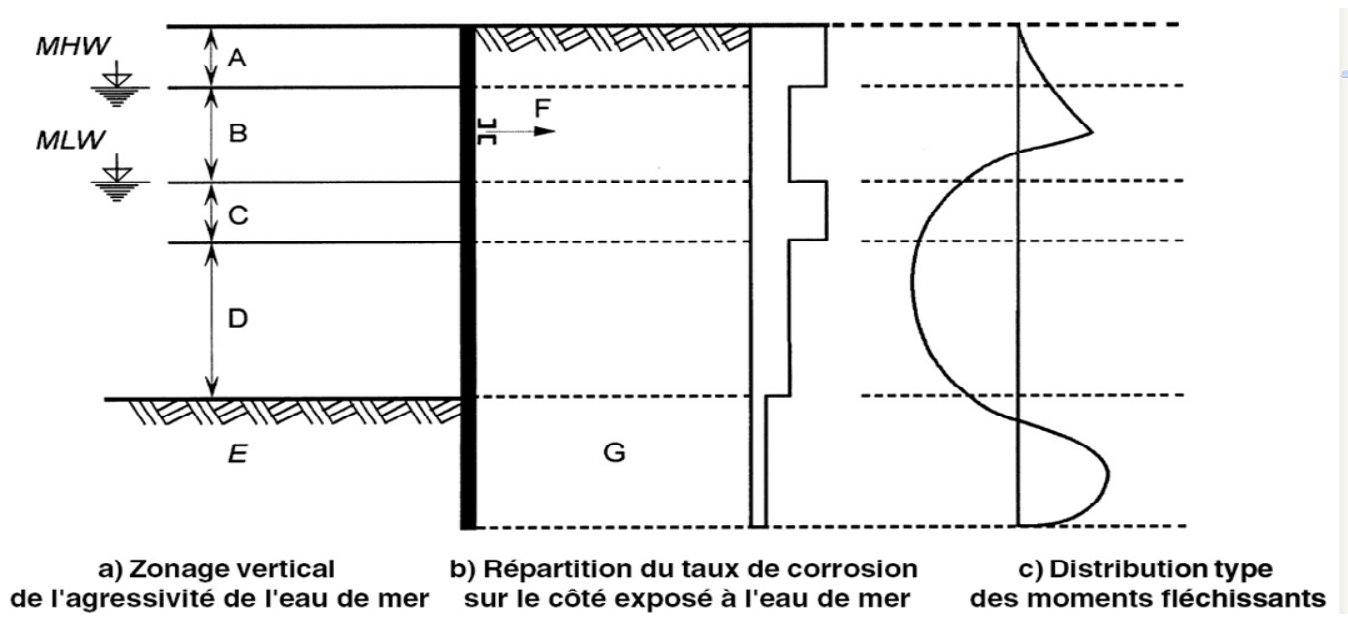

Figure 2. Zones d'exposition et moment fléchissant.

Selon les zones d'exposition, les caractéristiques physico-chimique du milieu et les conditions de contacts, les mécanismes de la corrosion (amorçage, cinétique et volumes ou surfaces affectés) peuvent être de différents types et présenter des aspects de dégradations très caractéristiques (généralisés ou localisés, en surface ou pérforante) CEFRACOR (2006) :

a) Zone atmosphérique : Corrosion généralisée en surface.

b) Zone d'embruns : Corrosion localisée avec parties de surfaces fortement détériorées (feuilletage, crevasses, cratères, perforations). Cette corrosion sévère que subit l'acier dans la zone des éclaboussures affecte les têtes de pieux et la jonction pieux chevêtres. La perforation à ce niveau peut atteindre les armatures des talons en BA.

c) Zone de marnage : Combinaison de mécanismes de corrosion atmosphérique partielle (temps d'exposition à l'air et d'immersion alternés) et d'action mécanique. Dans cette zone on peut rencontrer : de l'érosion par l'action des vagues, exacerbée pendant les périodes de grosse mer, de l'abrasion sous l'action des débris flottants, des armatures et des navires à l'accostage, de la prolifération de salissures marines et de la contamination éventuelle de l'eau par des films d'huile. Ces paramètres peuvent être favorables à la corrosion dans certains cas.

d) Zone des plus basses eaux : Corrosion par aération différentielle. La zone de marnage est oxygénée et constitue une cathode, la zone immergée juste en dessous constitue une anode soumise à une corrosion accélérée.

e) Zone de transition partie immergée/enterrée : Risque de corrosion bactérienne.

f) Zone de consolidation d'une structure métallique souvent siège de corrosion galvanique. Il est très rare que les métaux de consolidation soient de même composition métallurgique que celle de la structure à consolider. 
Autant le découpage en objets de visites que l'examen de l'état structurel (ex. : mesures d'épaisseurs résiduelles par ultra son) sont déterminés par la prise en compte de ces différentes zones ainsi définies pour un site portuaire donné. L'expertise en construction métallique et en corrosion sont requise pour mener à bien ce type de visite VSC dans le cas de structures métalliques en mer. Cet exemple met en exergue la nécessité de réaliser le bon échantillonnage pour réussir une inspection sur les zones critiques et cerner ainsi l'importance des pertes d'épaisseurs et qualifier l'état des composants de la structure.

La dégradation en tête de pieux (liaisonné avec une poutre en BA) ou en zone de basses eaux (moment fléchissant important) démontre la criticité de ces zones et oriente vers une vigilance et une surveillance régulière d'une part et un échantillonnage des mesures plus concentrées au détriment d'autres zones moins critiques.

Dans le cas de la protection contre la corrosion des structures métalliques, l'approche VSC est appropriée pour optimiser les interventions de surveillance, vérifications et mesures sur des surfaces aussi importantes que celles d'un nombre élevé des pieux sous les quais ou des palplanches d'un long rideau.

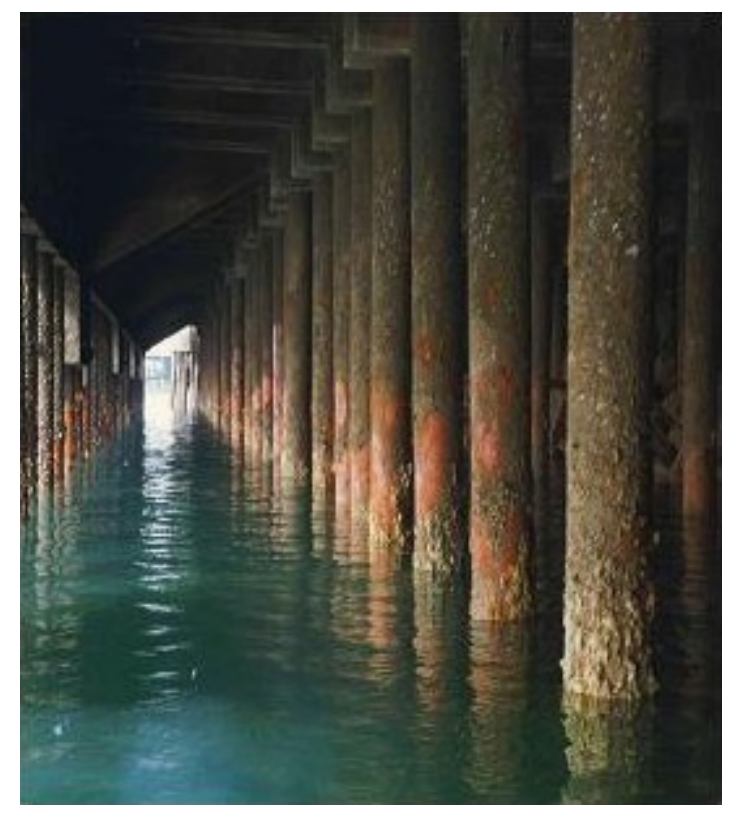

Figure 3. Pieux et corrosion.

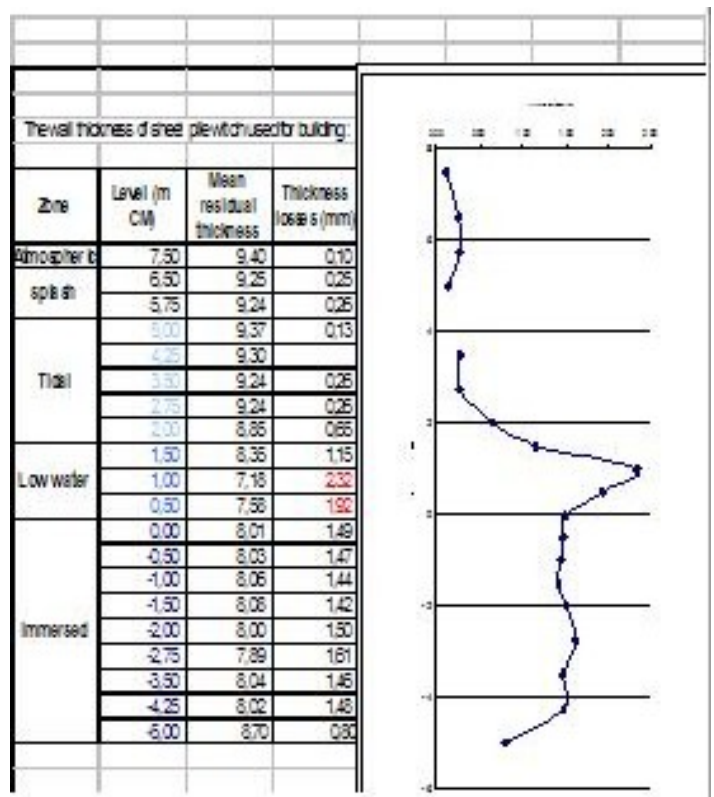

Figure 4. Mesures d'épaisseur en zone critique.

\section{Conclusions}

Prédire la durée de vie résiduelle d'un ouvrage donné n'est pas chose facile et c'est la principale demande des gestionnaires. Cette prévision dépend de l'état actuel (souvent difficile à cerner) et de la cinétique du vieillissement, elle même fonction des caractéristiques du matériau et des agressions du milieu. Evaluer les propriétés 


\section{XII ${ }^{\text {èmes }}$ Journées Nationales Génie Côtier - Génie Civil \\ Cherbourg, 12-14 juin 2012}

matérielles et géométriques de l'ouvrage est donc un préalable indispensable à la prédiction de sa durée de vie. Dans le cas le plus fréquent pour les ouvrages portuaires, celui de l'altération des aciers par la corrosion, dans une exposition à un environnement donné, il faut connaître plusieurs paramètres de la corrosion selon le milieu en contact pour être capable d'estimer la durée de vie résiduelle.

C'est l'ensemble de ces connaissances (électrochimique et chimique) ajoutées à celles structurelles (physiques et mécaniques) qui constitue l'enjeu des visites de type VSC.

La réussite de VSC repose sur :

a) la maîtrise de l'inventaire du parc et des utilisations ainsi que les choix stratégique du gestionnaire pour mieux cerner le périmètre des visites et hiérarchiser rationnellement les interventions dans un cadre économique donné.

b) La connaissance des structures composant l'ouvrage et leur fonctionnement, le matériau constitutif de ces structures et les interactions avec le milieu en contact, pour orienter les visites, cibler les diagnostics et réussir à collecter des informations pertinentes pour une estimation de l'état structurel plus crédible.

c) La traçabilité des informations collectées et des interventions réalisées pour mieux suivre les dérives éventuelles et corriger l'estimation de la durée de vie résiduelle.

La VSC peut donner de bons résultats grâce au travail coordonné entre le gestionnaire qui définit sa stratégie pour l'utilisation des ouvrages et ses priorités d'une part et à la compétence du chargé des visites (l'expert du domaine) qui réalise les évaluations des état des structures en opérant rationnellement de manière à limiter les interventions au juste nécessaire tout en recherchant la crédibilité des données d'autre part.

\section{Références bibliographiques}

AFGC (2003). Application des notions de fiabilité à la gestion des ouvrages existants. Sous la direction Christian CREMONA.

AFGC (2005). Méthodologie d'évaluation non destructive de l'état d'altération des ouvrages en béton armé. Sous la direction Denys BREYSSE et Odile ABRAHAM.

CEFRACOR (2006). La corrosion des structures métalliques en site portuaires. Brahim BENAISSA. Journée Aix en Pce.

CETMEF (2011). Application de la VSC à un parc d'ouvrages portuaires.

LCPC (2006) VSC Méthode d'aide à la gestion de patrimoines. Guide technique, Collection Techniques et Méthodes des laboratoires des ponts et chaussées, Août 2006. 
Thème 4-Ouvrages portuaires et offshore 